\title{
Effects of convection and diffusion of the vapour in evaporating liquid films
}

\author{
Kentaro Kanatani ${ }^{1 \dagger}$ \\ ${ }^{1}$ Faculty of Engineering, Yokohama National University, \\ 79-5 Tokiwadai, Hodogaya, Yokohama 240-8501, Japan \\ (Received ?; revised ?; accepted ?. - To be entered by editorial office)
}

We propose a novel model of a pure liquid film evaporating into an inert gas, taking into account an effect of convection of the vapour by the evaporation flow of the gas. For the liquid phase, the long-wave approximation is applied to the governing equations. Assuming that fluctuations of the vapour concentration in the gas phase are localised in the vicinity of the liquid-gas interface, we consider only the limit of the mass transport equation at the interface. The diffusion term in the vertical direction of the mass transport equation is modelled by introducing the concentration boundary layer above the liquid film and solving the stationary convection-diffusion equation for the concentration inside the boundary layer. We investigate the linear stability of a flat film based on our model. The effect of vapour diffusion along the interface mitigates the Marangoni effect for short-wavelength disturbances. The local variation of vertical advection is found to be negligible. A critical thickness above which the film is stable exists under the presence of gravity. The effect of fluctuation of mass loss of the liquid induced by horizontal vapour diffusion becomes the primary instability mechanism in a very thin region. The effects of the resistance of phase change and the time derivative of the interface concentration are also examined.

\section{Key words:}

\section{Introduction}

Evaporation or condensation is encountered in many practical situations. Because of its importance in technological applications, a number of works are dedicated to the stability of an evaporating liquid layer and subsequent pattern formation both experimentally (Mancini \& Maza 2004; Kimball et al. 2012; Chauvet et al. 2012) and theoretically (Palmer 1976; Merkt \& Bestehorn 2003; Haut \& Colinet 2005; Bestehorn 2007). Here the instability often involves the Marangoni effect, which is caused by the dependence of the liquid-gas surface tension on the temperature. For an evaporating liquid layer in contact only with its own vapour, the Marangoni effect can hardly appear because the interface temperature is almost constant from the condition of local thermodynamic equilibrium, which is a reasonable approximation in most cases. On the other hand, when an inert gas is present in the gas phase, the Marangoni effect arises from fluctuations of partial pressure of the volatile component.

If the liquid layer is sufficiently thick, the instability results in convection of the temperature. Here the liquid-gas interface is almost flat. As the liquid layer becomes thinner, the convective state of the temperature is changed to the conductive state. In the latter

$\dagger$ Email address for correspondence: kentaro@ynu.ac.jp 
case, the instability occurs against the flat interface. Owing to the effect of the surface tension, the deformation of the interface manifests itself as the long-wavelength deviation from the flat state. The stability and nonlinear dynamics of such two-dimensional evaporating or condensing liquid films were investigated by Burelbach et al. (1988) using the long-wave theory. Subsequently, three-dimensional patterns of evaporating liquid films were numerically studied in several situations (Oron 2000; Schwartz et al. 2001; Bestehorn \& Merkt 2006; Bestehorn 2007) in the framework of the long-wave theory. However, in all of these long-wave models of evaporating liquid films, the effect of the local variation of the vapour concentration was not taken into account. The presence of this effect allows fluctuations of the interface temperature and hence the emergence of the Marangoni effect.

Sultan et al. (2005) considered static diffusion of the vapour above an evaporating liquid film. In their model, the distribution of the vapour concentration obeys the Laplace equation, which is incorporated into the long-wave evolution equation of the film. Any convection effects of the vapour were disregarded assuming slow evaporation (the same assumption was also employed in Haut \& Colinet (2005)). Nevertheless, in this paper we question the neglect of advection of the vapour in the problem of evaporating thin liquid films. In order to compare the effects of convection and diffusion, we quantify the speeds of evaporation and diffusion. First, the evaporation speed of the gas at the liquid-gas interface is represented by $v_{\mathrm{ev}}=J / \rho_{g}$ from the mass balance condition where $J$ is the evaporation mass flux and $\rho_{g}$ the density of the gas (note that in Sultan et al. (2005) the liquid density is used instead of the gas density in the definition of the evaporation speed, which therefore means that of the interface). Second, the diffusion speeds in the horizontal and vertical directions are $D \partial_{x} c$ and $D \partial_{z} c$ where $D$ denotes the mass diffusivity of the gas, $c$ the mass concentration of the volatile component, and $\partial_{x}$ and $\partial_{z}$ the partial differential operators with respect to the horizontal and vertical directions. If we assume local thermodynamic equilibrium at the liquid-gas interface, the vapour concentration at the interface is a function of the interface temperature $T$ through the partial pressure of the volatile component $p_{v}$. Then, the horizontal derivative of the vapour concentration at the interface is expressed by

$$
\partial_{x} c=\frac{d c}{d p_{v}} \frac{d p_{v}}{d T} \partial_{x} T .
$$

Here, $d p_{v} / d T$ is defined as the slope of the coexistence curve of the volatile component on the pressure-temperature diagram, and given by the Clausius-Clapeyron relation

$$
\frac{d p_{v}}{d T}=\frac{\rho_{v} \rho L}{\left(\rho-\rho_{v}\right) T} \simeq \frac{\rho_{v} L}{T},
$$

where $\rho_{v}$ is the density of the volatile component in the gas, which is much less than the liquid density $\rho$, and $L$ the latent heat of vaporisation. If the inert and volatile components of the gas may be regarded as ideal gases, the ratio of the vapour partial pressure to the total pressure $p_{0}$ is equal to the molar fraction of the volatile component $X_{v}$. Since the mass concentration is related with the molar fraction by

$$
c=\frac{M_{v} X_{v}}{M_{v} X_{v}+M_{i}\left(1-X_{v}\right)},
$$

where $M_{i}$ and $M_{v}$ are the molar masses of the inert and volatile components, the above condition is written by

$$
\frac{p_{v}}{p_{0}}=X_{v}=\frac{M_{i} c}{M_{v}+\left(M_{i}-M_{v}\right) c} .
$$




$$
\begin{array}{ll}
\eta=10^{-3} \mathrm{~kg} /(\mathrm{m} \mathrm{s}) & \lambda=0.182 \mathrm{~W} /(\mathrm{K} \mathrm{m}) \\
\rho=789 \mathrm{~kg} / \mathrm{m}^{3} & L=878 \times 10^{3} \mathrm{~J} / \mathrm{kg} \\
\sigma_{0}=22.8 \times 10^{-3} \mathrm{~N} / \mathrm{m} & \gamma=8.4 \times 10^{-5} \mathrm{~N} /(\mathrm{m} \mathrm{K}) \\
D=10^{-5} \mathrm{~m}^{2} / \mathrm{s} & p_{0}=101 \times 10^{3} \mathrm{~N} / \mathrm{m}^{2}
\end{array}
$$

TABLE 1. Physical properties used for the ethanol-nitrogen system (Haut \& Colinet 2005). The value of the surface tension is taken from Dittmar et al. (2003).

As earlier mentioned, the temperature inside the liquid is in the conductive state for sufficiently thin films. This fact suggests that the temperature gradient in the vertical direction is constant and that the interface temperature depends on the film thickness $h$. Thus, the temperature derivative along the interface is roughly estimated as

$$
\partial_{x} T \simeq \partial_{z} T \partial_{x} h \simeq \partial_{z} T \frac{h}{l}
$$

where $l$ is the characteristic lateral length scale of the liquid film. Note that in (1.5) the effect of the vapour diffusion along the interface was not considered; if this effect is taken into account, $\left|\partial_{x} T\right|$ and consequently $\left|\partial_{x} c\right|$ become smaller. For a nearly flat film the heat flux across the liquid balances with the latent heat,

$$
\lambda\left|\partial_{z} T\right| \simeq J L,
$$

where $\lambda$ is the thermal conductivity of the liquid. Substituting (1.2), (1.4), (1.5), (1.6) and the expressions for the densities of ideal gases

$$
\rho_{g}=\frac{M_{i} M_{v} p_{0}}{\left\{M_{v}+\left(M_{i}-M_{v}\right) c\right\} R T}, \quad \rho_{v}=c \rho_{g},
$$

where $R$ is the universal gas constant, one finds that the ratio between the evaporation and horizontal diffusion speeds does not depend on the evaporation mass flux:

$$
\frac{\left|D \partial_{x} c\right|}{\left|v_{\mathrm{ev}}\right|} \simeq \frac{M_{i} M_{v} c p_{0} D L^{2} h}{R^{2} T^{3} \lambda l}=c\left(\frac{T_{0}}{T}\right)^{3} \frac{h}{l} .
$$

Note that $T_{0}$ is determined solely by the physical properties of the system. For instance, we take the ethanol-nitrogen system (Haut \& Colinet 2005). Using the data shown in table $1, T_{0} \simeq 430 \mathrm{~K}$ and therefore $\left(T_{0} / T\right)^{3} \simeq 3$ at $T=300 \mathrm{~K}$. Allowing for the fact that the aspect ratio of the liquid film $h / l$ is normally $10^{-1}$ to $10^{-2}$ and $c<1$, it follows that the speed of evaporation is much larger than that of the vapour diffusion along the interface at least for this system. As mentioned after (1.5), the ratio of the speeds (1.8) further decreases by including the effect of the horizontal vapour diffusion. Regarding the vertical diffusion, from the mass balance of one component at the liquid-gas interface the ratio between the evaporation and vertical diffusion speeds is given by

$$
\frac{\left|D \partial_{z} c\right|}{\left|v_{\mathrm{ev}}\right|}=1-c
$$

which is exact for a flat interface. Thus, the speed of vertical diffusion cannot exceed that of evaporation at least near the interface, regardless of the evaporation rate and even the liquid layer depth.

For the reason described above, this paper aims to construct a theory for a pure liquid film evaporating into an inert gas, taking into account an effect of convection of the vapour by the evaporation flow of the gas. For the liquid phase, we shall apply the standard 
long-wave approximation (Oron et al. 1997). The situation is much more complicated in the gas phase, because the mass transport equation includes the gas velocity, which cannot be known without solving the bulk equations of the gas phase simultaneously. However, in order to simplify the problem we shall assume that fluctuations of the vapour concentration due to the deformation of the liquid-gas interface are restricted to the vicinity of the interface. This allows us the description where the vapour concentration two-dimensionally varies along the interface. As a result, we only have to consider the limit of the mass transport equation at the interface on the gas side. The gas velocity and vapour concentration gradient in the vertical direction at the interface, included in the advection term of the mass transport equation, are expressed in terms of the evaporation mass flux using the conservation laws of the total mass and that of one component at the interface. Only the vertical diffusion term among those in the mass transport equation cannot be described by quantities at the interface. In order to model this term, we introduce the concentration boundary layer above the liquid film, on the top of which the vapour concentration is fixed at a constant value. This approach is analogous to that of Haut \& Colinet (2005). However, they considered also the viscous and thermal boundary layers, which are irrelevant in this work (we assume that the heat flux in the gas phase is much smaller than that due to latent heat). Since the concentration distribution inside the boundary layer can be described by the stationary convection-diffusion equation, we obtain the expression for the vertical diffusion term at the interface in the mass transport equation from the solution of the stationary convection-diffusion equation. Then, we assume that this expression holds even when the interface is deformed. Actually, this expression becomes exact in the long-wavelength limit, where the characteristic lateral length scale is sufficiently larger than the boundary layer depth to neglect the effects of concentration and momentum diffusion in the horizontal direction in the gas phase. The long-wave equation for the liquid and the mass transport equation at the interface on the gas side compose a closed system for the two unknown variables, the film thickness and the vapour concentration at the interface.

The effect of mass loss of the liquid often influences the linear stability property of an evaporating flat surface (Palmer 1976; Burelbach et al. 1988; Bestehorn \& Merkt 2006; Bestehorn 2007; Thiele 2010). For simplicity we adopt the frozen-time approach, where the linear stability analysis is carried out against the "hypothetically motionless" interface at each instance or film thickness (of course in reality the interface is moving owing to evaporation). If the effect of mass loss on the growth rate of disturbances is independent of their wavenumber, which is the case for the one component gas, the dispersion curve is simply shifted upward in the framework of the frozen-time approach (see e.g. figure 19 of Burelbach et al. (1988)). However, in the present case the effect of vapour diffusion along the interface affects the evaporation mass flux through the local thermodynamic equilibrium condition, and is stronger for short wavelength disturbances. Thus, the effect of mass loss is wavenumber-dependent under the presence of an inert gas, and changes the shape of the dispersion curve and the maximum growth wavenumber.

In the current paper, only the evaporating case is addressed. However, our model is also applicable to condensing liquid films. In this case, thermocapillarity has a stabilising effect unless the liquid-gas surface tension increases as the temperature increases. Therefore, the Marangoni instability is usually not expected for a condensing pure liquid film. The situation becomes different, nevertheless, when the liquid consists of two or more components. If the concentration of one component which has a stronger surface tension than those of the other components becomes larger for higher temperatures, solutocapillarity destabilises the liquid film. This phenomenon is known as the Marangoni dropwise condensation (Mirkovich \& Missen 1961), and was recently modelled by Kanatani (2013). 


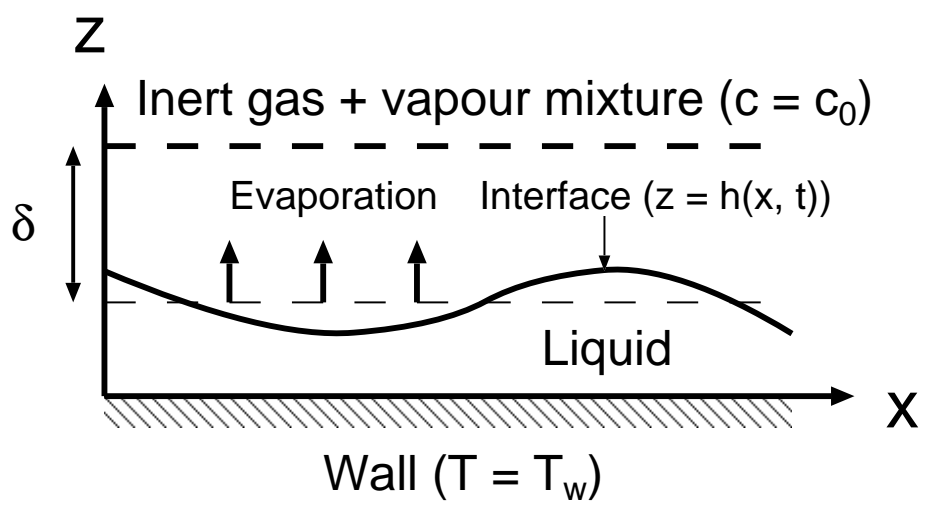

FIGURE 1. Sketch of the studied system.

In the model of Kanatani (2013), the effect of concentration diffusion along the liquidvapour interface was not taken into account. The inclusion of this effect as in the present model is a future work.

This paper is organised as follows. First the governing equations and boundary conditions are presented in the dimensional form in $§ 2.1$. Here, the two-dimensional (actually one-dimensional) limit of the mass transport equation at the interface on the gas side is also derived. Dimensionless parameters of this system are introduced in $\S 2.2$. The nondimensionalised model equations are shown in $\S 2.3$. The basic state of the model, whose stability is investigated in the following section, is obtained in $\S 3$. Linear stability results are given in $\S 4$. Because of the complexity of the equation for the growth rate, we consider four cases in order. First the effects of mass loss, the resistance of phase change, and the time derivative of the interface concentration are neglected in $\S 4.1$. Second the effect of mass loss is incorporated in $§ 4.2$. The effect of departure from local thermodynamic equilibrium is examined in $\$ 4.3$. In addition we briefly discuss the effect of the time derivative of the interface concentration in $\$ 4.4$. Concluding remarks are drawn in $\S 5$.

\section{Formulation}

\subsection{Governing equations}

We consider a two-dimensional thin liquid film on a horizontal wall at a temperature $T_{w}$, as depicted in figure 1 . The $x$-axis coincides with the liquid-solid boundary and the $z$-axis points vertically upward to the wall. The mass fraction of the surrounding vapour is fixed to $c_{0}$ at some distance $\delta$ from the liquid film; here $\delta$ is assumed to be much larger than the thickness of the film $h$ so that the former is never affected by the variation of the latter. The governing equations are the continuity, Navier-Stokes and energy equations in the liquid phase and the mass transport equation in the gas phase:

$$
\begin{aligned}
& \boldsymbol{\nabla} \cdot \boldsymbol{v}=0, \\
& \rho\left(\partial_{t} \boldsymbol{v}+\boldsymbol{v} \cdot \boldsymbol{\nabla} \boldsymbol{v}\right)=-\boldsymbol{\nabla} p+\eta \nabla^{2} \boldsymbol{v}-\rho g \boldsymbol{e}_{z}, \\
& \partial_{t} T+\boldsymbol{v} \cdot \boldsymbol{\nabla} T=\kappa \nabla^{2} T, \\
& \partial_{t} c+\boldsymbol{v}_{g} \cdot \boldsymbol{\nabla} c=D \nabla^{2} c,
\end{aligned}
$$

where $\boldsymbol{v}=(u, w), p$, and $T$ are the velocity, pressure, and temperature fields in the liquid, respectively; $c$ and $\boldsymbol{v}_{g}=\left(u_{g}, w_{g}\right)$ the mass concentration of the volatile component and 
velocity in the gas. The differential operator is $\boldsymbol{\nabla} \equiv\left(\partial_{x}, \partial_{z}\right)$. The symbols $\rho, \eta$, and $\kappa$ denote the density, dynamic viscosity, and thermal diffusivity of the liquid, respectively; $D$ the mass diffusivity of the gas, all assumed to be constant, $g$ the gravitational acceleration, and $\boldsymbol{e}_{z}$ the unit vector in the $z$ direction.

We impose the no-slip, no-penetration and constant temperature boundary conditions at the wall,

$$
\boldsymbol{v}=\mathbf{0}, \quad T=T_{w} \quad \text { at } \quad z=0 .
$$

At the liquid-gas interface $(z=h(x, t))$, the mass balance condition is expressed as

$$
J=\rho\left(\boldsymbol{v} \cdot \boldsymbol{n}-\boldsymbol{v}_{I} \cdot \boldsymbol{n}\right)=\rho_{g}\left(\boldsymbol{v}_{g} \cdot \boldsymbol{n}-\boldsymbol{v}_{I} \cdot \boldsymbol{n}\right),
$$

where $J$ is the mass flux, $\boldsymbol{n}=\left(-\partial_{x} h, 1\right) / \sqrt{1+\left(\partial_{x} h\right)^{2}}$ the upward-directed unit vector normal to the interface, $\boldsymbol{v}_{I}$ the interface velocity, which satisfies the kinematic condition,

$$
\boldsymbol{v}_{I} \cdot \boldsymbol{n}=\frac{\partial_{t} h}{\sqrt{1+\left(\partial_{x} h\right)^{2}}},
$$

and $\rho_{g}$ the gas density. We assume the continuity of the tangential velocity along the interface, interfacial stress and energy:

$$
\begin{gathered}
\boldsymbol{v} \cdot \boldsymbol{t}=\boldsymbol{v}_{g} \cdot \boldsymbol{t}, \\
\left(p-p_{0}\right) \boldsymbol{n}-2 \eta E \cdot \boldsymbol{n}+2 \sigma H \boldsymbol{n}+(\boldsymbol{t} \cdot \boldsymbol{\nabla} \sigma) \boldsymbol{t}=\mathbf{0}, \\
J L+\lambda \boldsymbol{\nabla} T \cdot \boldsymbol{n}=0,
\end{gathered}
$$

where $\boldsymbol{t}=\left(1, \partial_{x} h\right) / \sqrt{1+\left(\partial_{x} h\right)^{2}}$ the unit tangent vector to the interface, $p_{0}$ is the ambient gas pressure, $E$ the rate-of-strain tensor of the liquid, $\sigma$ the surface tension, $H$ the mean curvature of the interface,

$$
2 H=\frac{\partial_{x x} h}{\left\{1+\left(\partial_{x} h\right)^{2}\right\}^{3 / 2}},
$$

$L$ the latent heat and $\lambda$ the thermal conductivity of the liquid. Note that the effects of vapour recoil, stress induced by the vapour motion, heat flux in the gas phase and viscous dissipation have been neglected in (2.9) and (2.10) assuming slow evaporation. For rapidly evaporating liquids, the above effects except that of the gas heat flux become significant (see Palmer 1976). However, for thin films the effects of the vapour stress and viscous dissipation are neglected under the long-wave approximation (see Burelbach et al. 1988), unless there is an internal or external flow of the gas in the lateral direction, which causes a shear stress on the interface. The former case was considered by Kanatani (2010) and Kanatani \& Oron (2011), where the lateral vapour flow arises from the confined geometry of the gas phase. The latter case has been recently addressed in several works (e.g. Scheid et al. 2012; Liu \& Kabov 2012, and references therein). Regarding the surface tension, we use the linear approximation for the dependence on the interface temperature $T_{I}$ :

$$
\sigma=\sigma_{0}-\gamma\left(T_{I}-T_{w}\right) .
$$

Furthermore, the mass flux balance of the volatile component reads

$$
J=-\rho_{g} D \nabla c \cdot \boldsymbol{n}+c J .
$$

The second term on the right hand side of this equation, which corresponds to advection of the volatile component by the evaporation gas flow, was disregarded in the study of 
Sultan et al. (2005). Finally, we use the standard Hertz-Knudsen law,

$$
J=\alpha\left\{p_{s}(T)-p_{v}\right\} \sqrt{\frac{M_{v}}{2 \pi R T}},
$$

where $p_{s}$ is the saturation pressure of the liquid, $p_{v}$ the partial pressure of the volatile component in the gas, $M_{v}$ the molar mass of the volatile component, $R$ the universal gas constant, and $\alpha(<1)$ the accommodation coefficient. In this study, we use a linearised expression of (2.14) with respect to temperature and concentration. First we set the reference temperature as the wall temperature and the reference concentration $c_{w}$ such that

$$
\frac{p_{s}\left(T_{w}\right)}{p_{0}}=\frac{M_{i} c_{w}}{M_{v}-\left(M_{v}-M_{i}\right) c_{w}},
$$

i.e., thermodynamic equilibrium holds at $T_{w}$ and $c_{w}$ (cf. equation (1.4)). Hereafter we refer to $c_{w}$ as "wall concentration". Using equations (1.2), (1.4), and (1.7), the saturation and partial pressures are linearised around $T_{w}$ and $c_{w}$ as

$$
\begin{aligned}
p_{s}\left(T_{I}\right) & =p_{s}\left(T_{w}\right)+\frac{M_{v} p_{s}\left(T_{w}\right) L}{R T_{w}^{2}}\left(T_{I}-T_{w}\right), \\
p_{v} & =p_{s}\left(T_{w}\right)+\frac{M_{v} M_{i} p_{0}}{\left\{M_{v}-\left(M_{v}-M_{i}\right) c_{w}\right\}^{2}}\left(c_{I}-c_{w}\right),
\end{aligned}
$$

where $c_{I}$ the vapour concentration at the interface. Substitution of (2.16) and (2.17) into (2.14) yields

$$
J=\alpha\left[\frac{M_{v} p_{s}\left(T_{w}\right) L}{R T_{w}^{2}}\left(T_{I}-T_{w}\right)-\frac{M_{v} M_{i} p_{0}}{\left\{M_{v}-\left(M_{v}-M_{i}\right) c_{w}\right\}^{2}}\left(c_{I}-c_{w}\right)\right] \sqrt{\frac{M_{v}}{2 \pi R T_{w}}} .
$$

Here we have neglected the temperature dependence of the proportionality coefficient in (2.14).

As stated in $\S 1$, we consider only the limit $z \rightarrow h(x, t)$ of the mass transport equation in the gas phase (2.4). From the boundary conditions (2.6) and (2.13) the gas velocity and vapour concentration gradient normal to the interface are represented by

$$
\boldsymbol{v}_{g} \cdot \boldsymbol{n}=\frac{J}{\rho_{g}}, \quad \boldsymbol{\nabla} c \cdot \boldsymbol{n}=-\frac{(1-c) J}{\rho_{g} D} \quad \text { at } \quad z=h,
$$

where we have assumed that the gas velocity is much larger than the interface velocity. Substituting (2.19) together with (2.8) into (2.4) after taking the limit $z \rightarrow h$, we obtain

$$
\partial_{t} c_{I}+u_{I} \partial_{x} c_{I}-\frac{\left(1-c_{I}\right) J^{2}}{\rho_{g}^{2} D}=D\left(\partial_{x x} c_{I}+\left.\partial_{z z} c\right|_{z=h}\right)
$$

where $u_{I}(x, t)=u(x, z=h(x, t), t)$ is the horizontal component of the liquid velocity at the interface. Note that in (2.20) the small inclination of the interface has been assumed $\left(\partial_{x} h \ll 1\right)$.

In order to close the system we need to model the second derivative of the concentration with respect to $z$ in (2.20). As outlined in $\S 1$, we first consider the concentration field inside the boundary layer at the flat-film state, where all the variables do not depend on the horizontal coordinate $x$. Then the mass transport equation reduces to the onedimensional stationary convection-diffusion equation in the vertical direction,

$$
w_{g} \partial_{z} c=D \partial_{z z} c
$$

Here the temporal variation of the concentration has been neglected, given that the 
concentration at the liquid-gas interface can be regarded as constant during the characteristic time in the gas phase. Furthermore, the gas velocity $w_{g}$ is constant in the vertical direction from the continuity equation. With the boundary conditions $c=c_{I}$ at $z=h$ and $c=c_{0}$ at $z=h+\delta$ the solution of (2.21) is expressed by

$$
c=c_{0}+\left(c_{I}-c_{0}\right) \frac{1-e^{-w_{g}(h+\delta-z) / D}}{1-e^{-w_{g} \delta / D}} .
$$

Since the gas velocity $w_{g}$ is related with the mass flux through $J=\rho_{g} w_{g}$, the expression for $w_{g}$ is obtained from (2.13) using (2.22) as

$$
w_{g}=\frac{D}{\delta} \log \left(\frac{1-c_{0}}{1-c_{I}}\right) .
$$

Hence the second derivative of the concentration at the interface becomes

$$
\left.\partial_{z z} c\right|_{z=h}=-\left(1-c_{I}\right)\left\{\frac{1}{\delta} \log \left(\frac{1-c_{0}}{1-c_{I}}\right)\right\}^{2} .
$$

We assume that this equation holds even when the interface is deformed. This assumption is valid as long as the gas velocity $w_{g}$ can be regarded as vertically (not horizontally) constant, which follows from the neglect of the horizontal variation of the horizontal component of the gas velocity in the continuity equation. Therefore, equations (2.21), $(2.22),(2.23)$, and $(2.24)$ are exact in the limit of the long-scale variation $\left(\partial_{x} \rightarrow 0\right)$, where the boundary layer of the gas is thin enough compared to the characteristic lateral length scale of the system to neglect momentum diffusion in the horizontal direction. Then substitution of (2.24) into (2.20) gives

$$
\frac{\partial_{t} c_{I}+u_{I} \partial_{x} c_{I}}{D}-\partial_{x x} c_{I}=\left(1-c_{I}\right)\left[\left(\frac{J}{\rho_{g} D}\right)^{2}-\left\{\frac{1}{\delta} \log \left(\frac{1-c_{0}}{1-c_{I}}\right)\right\}^{2}\right]
$$

\subsection{Nondimensionalisation}

We scale lengths, time, velocities and pressure by $d, d^{2} \rho / \eta, \eta /(d \rho)$ and $\eta^{2} /\left(d^{2} \rho\right)$, respectively, where $d$ is the characteristic film thickness. Note that the film thickness decreases during evaporation. However, $d$ is defined as a proper constant and never varies throughout the temporal evolution of the liquid film. From (2.25) we shall choose the mass flux scale as $\rho_{g} D / d$. Then it is natural from (2.10) to nondimensionalise temperature by $L \rho_{g} D / \lambda$. We set the dimensionless pressure and temperature to 0 at $p_{0}$ and $T_{w}$. The above nondimensionalisation yields the following dimensionless parameters:

$$
\begin{aligned}
& G=\frac{g d^{3} \rho^{2}}{\eta^{2}}, \quad S=\frac{\sigma_{0} d \rho}{\eta^{2}}, \quad E=\frac{\rho_{g} D}{\eta}, \quad M a=\frac{d \rho L \rho_{g} D \gamma}{\eta^{2} \lambda}, \quad N=\frac{\eta}{\rho D}, \quad \Delta=\frac{\delta}{d}, \\
& Q=\frac{\lambda R T_{w}^{2}}{\alpha d M_{v} p_{s}\left(T_{w}\right) L^{2}} \sqrt{\frac{2 \pi R T_{w}}{M_{v}}}, \quad \Pi=\frac{M_{i} p_{0} \lambda R T_{w}^{2}}{\left\{M_{v}-\left(M_{v}-M_{i}\right) c_{w}\right\}^{2} \rho_{g} D p_{s}\left(T_{w}\right) L^{2}} .
\end{aligned}
$$

Here $G, S, E$, and $M a$ characterise the effects of gravity, surface tension, mass loss, and thermocapillarity, respectively; $N$ denotes the Schmidt number of the gas multiplied by the kinematic viscosity ratio between the liquid and gas; $\Delta$ is the ratio between the thicknesses of the gas and liquid; $Q$ and $\Pi$ represent the resistance to phase change and the slope of the coexistence curve between temperature and concentration.

Note that the boundary layer depth $\delta$ is a quantity difficult to measure in experiments. For this reason it is important to express $\delta$ in terms of experimentally measurable quantities. In our case the heat flux on the wall $q_{w}=-\left.\lambda \partial_{z} T\right|_{z=0}$ is one of the measurable 
quantities. Since for the quasi-steady state the energy equation (2.3) reduces to $\partial_{z z} T=0$, the vertical temperature gradient is constant inside the liquid for a flat film. Then the boundary condition (2.10) and the governing equation (2.25) become

$$
q_{w}=J L, \quad J=\frac{\rho_{g} D}{\delta} \log \left(\frac{1-c_{0}}{1-c_{I}}\right) .
$$

Eliminating $J$ from the above equations and approximating $c_{I}$ by $c_{w}, \delta$ can be defined with $q_{w}$ :

$$
\delta=\frac{\rho_{g} D L}{q_{w}} \log \left(\frac{1-c_{0}}{1-c_{w}}\right) .
$$

Hence, if the heat flux at the liquid-solid boundary in the flat-film state is measured from an experiment, one can determine the value of $\delta$ from (2.28).

\subsection{Model equations}

Assuming that the characteristic wavelength in the horizontal direction is much larger than the film thickness, we apply the long-wave approximation to the liquid film. Since the derivation of an evolution equation of the film thickness is straightforward, we shall omit it in this paper. For details the reader should refer to, for example, Oron et al. (1997). The dimensionless equation for the film thickness is derived as

$$
\partial_{t} h=\partial_{x}\left\{\frac{h^{3}}{3} \partial_{x}\left(G h-S \partial_{x x} h\right)+\frac{h^{2}}{2} M a \partial_{x} T_{I}\right\}-E J .
$$

The interface temperature follows from the solution of the energy equation (2.3) with the boundary conditions (2.5) and (2.10). Under the long-wave approximation

$$
T_{I}=-J h,
$$

which results from the linear temperature distribution with respect to the vertical coordinate. The dimensionless version of (2.18) is

$$
Q J=T_{I}-\Pi\left(c_{I}-c_{w}\right) .
$$

From (2.30) and (2.31) the mass flux can be written as

$$
J=\frac{\Pi\left(c_{w}-c_{I}\right)}{h+Q} .
$$

Substituting (2.30) and (2.32) into (2.29) and the nondimensionalised equation of (2.25), the final model equations become

$$
\begin{aligned}
\partial_{t} h=\partial_{x}\left[\frac{h^{3}}{3} \partial_{x}\left(G h-S \partial_{x x} h\right)-\right. & \left.\frac{M a \Pi h^{2}}{2} \partial_{x}\left\{\frac{h\left(c_{w}-c_{I}\right)}{h+Q}\right\}\right]-\frac{E \Pi\left(c_{w}-c_{I}\right)}{h+Q}, \\
N\left(\partial_{t} c_{I}+u_{I} \partial_{x} c_{I}\right)-\partial_{x x} c_{I}=\left(1-c_{I}\right) & \times\left[\left\{\frac{\Pi\left(c_{w}-c_{I}\right)}{h+Q}\right\}^{2}-\left\{\frac{1}{\Delta} \log \left(\frac{1-c_{0}}{1-c_{I}}\right)\right\}^{2}\right] .
\end{aligned}
$$

Here the interface velocity in (2.34) is calculated within the framework of the long-wave approximation:

$$
u_{I}=-\frac{h^{2}}{2} \partial_{x}\left(G h-S \partial_{x x} h\right)+M a \Pi h \partial_{x}\left\{\frac{h\left(c_{w}-c_{I}\right)}{h+Q}\right\},
$$

where (2.30) and (2.32) have been used. 


\section{Basic state}

First we take the flat-film state as the basic state $\left(\bar{h}(t), \bar{c}_{I}(t)\right)$, which is homogeneous in the horizontal direction. By dropping the partial derivatives with respect to $x$, the model equations (2.33) and (2.34) reduce to the following ordinary differential equations:

$$
\begin{aligned}
\frac{d \bar{h}}{d t} & =-\frac{E \Pi\left(c_{w}-\bar{c}_{I}\right)}{\bar{h}+Q} \\
\frac{d \bar{c}_{I}}{d t} & =\frac{1-\bar{c}_{I}}{N}\left[\left\{\frac{\Pi\left(c_{w}-\bar{c}_{I}\right)}{\bar{h}+Q}\right\}^{2}-\left\{\frac{1}{\Delta} \log \left(\frac{1-c_{0}}{1-\bar{c}_{I}}\right)\right\}^{2}\right] .
\end{aligned}
$$

For simplicity we neglect the time derivative of the vapour concentration at the interface in (3.2), which is justified later. Then equations (3.1) and (3.2) become

$$
\begin{aligned}
\frac{d \bar{h}}{d t} & =-\frac{E}{\Delta} \log \left(\frac{1-c_{0}}{1-\bar{c}_{I}}\right), \\
\bar{h} & =\frac{\Delta \Pi\left(c_{w}-\bar{c}_{I}\right)}{\log \left(\frac{1-c_{0}}{1-\bar{c}_{I}}\right)}-Q .
\end{aligned}
$$

By differentiating (3.4) with respect to time and substituting it into (3.3), we obtain an ordinary differential equation for $\bar{c}_{I}$ :

$$
\frac{d \bar{c}_{I}}{d t}=\frac{E}{\Delta^{2} \Pi} \frac{\left\{\log \left(\frac{1-c_{0}}{1-\bar{c}_{I}}\right)\right\}^{3}}{\frac{c_{w}-\bar{c}_{I}}{1-\bar{c}_{I}}+\log \left(\frac{1-c_{0}}{1-\bar{c}_{I}}\right)} .
$$

This equation is integrable using the method of separation of variables. The result is

$$
\left(1-c_{0}\right)\left[\operatorname{li} X-\frac{X}{\log X}+\frac{X-\frac{1-c_{w}}{1-c_{0}}}{(\log X)^{2}}\right]_{X=\left\{1-\bar{c}_{I}(t)\right\} /\left(1-c_{0}\right)}^{X=\left\{1-\bar{c}_{I}(0)\right\} /\left(1-c_{0}\right)}=\frac{2 E}{\Delta^{2} \Pi} t
$$

where

$$
\operatorname{li} X=\int_{0}^{X} \frac{d t}{\log t}
$$

is the logarithmic integral function and $[f(X)]_{X=b}^{X=a}=f(a)-f(b)$. Note that the solution (3.6) is valid even if the liquid layer is thick or condensing $\left(c_{0}>c_{w}\right)$. Nevertheless, if the variation of the vapour concentration is sufficiently small, we may linearise (3.3) and (3.4) around the wall concentration to yield

$$
\begin{aligned}
\frac{d \bar{h}}{d t} & =-\frac{E}{\Delta}\left\{1-\frac{\bar{h}+Q}{\Delta \Pi\left(1-c_{w}\right)}\right\} \log \left(\frac{1-c_{0}}{1-c_{w}}\right), \\
\bar{h} & =\frac{\Delta \Pi\left(c_{w}-\bar{c}_{I}\right)}{\log \left(\frac{1-c_{0}}{1-c_{w}}\right)}-Q .
\end{aligned}
$$

Then the temporal evolution of the basic film thickness is obtained as

$$
\bar{h}(t)=\Delta \Pi\left(1-c_{w}\right)-Q-\left\{\Delta \Pi\left(1-c_{w}\right)-\bar{h}(0)-Q\right\}\left(\frac{1-c_{0}}{1-c_{w}}\right)^{E t /\left\{\Delta^{2} \Pi\left(1-c_{w}\right)\right\}} .
$$




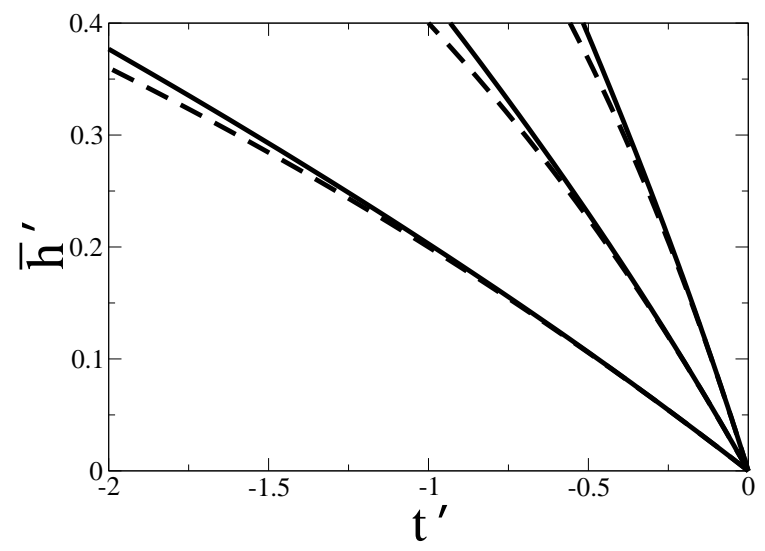

FiguRE 2. Temporal evolution of the basic film thickness in terms of the normalised variables (3.11). The solid curves have been calculated from (3.4) and (3.6). The dashed curves represent the linearised expression (3.12). $C^{-1}=0.8,0.6$, and 0.4 from left to right. Here we have set $\bar{h}^{\prime}(0)=0$.

Introducing new variables

$$
\bar{h}^{\prime}=\frac{\bar{h}+Q}{\Delta \Pi\left(1-c_{w}\right)}, \quad t^{\prime}=\frac{E t}{\Delta^{2} \Pi\left(1-c_{w}\right)}, \quad C=\frac{1-c_{0}}{1-c_{w}},
$$

equation (3.10) can be rewritten in the simple form

$$
\bar{h}^{\prime}\left(t^{\prime}\right)=1-\left(1-\bar{h}^{\prime}(0)\right) C^{t^{\prime}}
$$

Figure 2 compares the time evolution of the basic film thickness obtained from (3.4) and (3.6), and the linearised expression (3.12), in terms of the normalised variables (3.11) for different concentration ratios $C$. Here for all the curves the time has been set to zero when $\bar{h}^{\prime}$ vanishes (the disappearance time of the film for $Q=0$ ). For each $C$ the two curves show a good agreement for $\bar{h}^{\prime}<0.15$. The curves in figure 2 are convex, which implies increasing evaporation rate for decreasing film height. This is because the difference from the ambient and interface concentrations becomes large as the interface approaches the wall. In figure 4 of Haut \& Colinet (2005) the curves of the evolution of the liquid thickness are concave. This difference presumably arises from the fact that they kept the location where the concentration is fixed (the upper boundary) constant in time during their computation: in such a case, the decrease of the liquid depth increases the boundary layer depth and consequently decreases the evaporation rate.

In closing this section, the condition of negligible time derivative in (3.2) is obtained by comparing (3.2) and (3.5):

$$
\frac{E N}{\Pi} \frac{\log \left(\frac{1-c_{0}}{1-\bar{c}_{I}}\right)}{c_{w}-\bar{c}_{I}+\left(1-\bar{c}_{I}\right) \log \left(\frac{1-c_{0}}{1-\bar{c}_{I}}\right)} \ll 1,
$$

which is reasonable, unless $\bar{c}_{I}$ approaches one at fixed $c_{0}$, because the parameters $E$ and $N$ are usually much less than one. 


\section{Linear stability analysis}

In order to seek the stability property of the basic state, we superpose small disturbances of the normal mode

$$
\begin{aligned}
h(x, t) & =\bar{h}(t)+A \exp (i k x+\omega t), \\
c_{I}(x, t) & =\bar{c}_{I}(t)+B \exp (i k x+\omega t),
\end{aligned}
$$

with the wavenumber $k$ and the growth rate $\omega$. Note that since the basic state is timedependent, the exponent of the disturbances in (4.1) and (4.2) does not have an exact linear dependency on time; in other words $\omega$ is a function of time, which is written in the form of integration of some function of the basic solution (see Burelbach et al. 1988; Kanatani 2013). However, for simplicity we consider only the instantaneous growth rate at a given time or film thickness and regard $\omega$ as constant in time in the analysis below (the frozen-time approach). Substitution of (4.1) and (4.2) into the model equations (2.33) and (2.34) and linearisation with respect to the amplitudes of the disturbances $A$ and $B$ results in the following quadratic equation for $\omega$ :

$$
\begin{aligned}
&\left\{\omega+\frac{\bar{h}^{3} k^{2}}{3}\left(G+S k^{2}\right)-\frac{\Pi\left(c_{w}-\bar{c}_{I}\right)}{(\bar{h}+Q)^{2}}\left(E+\frac{M a Q \bar{h}^{2} k^{2}}{2}\right)\right\} \\
& \times\left\{N \omega+k^{2}+\frac{2 \Pi^{2}\left(1-\bar{c}_{I}\right)\left(c_{w}-\bar{c}_{I}\right)}{(\bar{h}+Q)^{2}}+\frac{2}{\Delta^{2}} \log \left(\frac{1-c_{0}}{1-\bar{c}_{I}}\right)\right\} \\
&-\frac{\Pi^{3}\left(1-\bar{c}_{I}\right)\left(c_{w}-\bar{c}_{I}\right)^{2}\left(M a \bar{h}^{3} k^{2}-2 E\right)}{(\bar{h}+Q)^{4}}=0 .
\end{aligned}
$$

Here, the variation of the factor $1-c_{I}$ in front of the square brackets in the right hand side of (2.34) has not been included, because the total value inside the square brackets in the basic state corresponds to the time derivative of the basic interface concentration from (3.2), which has been neglected in $\S 3$.

$$
\text { 4.1. Case } E=Q=N=0
$$

Because of the complexity of (4.3), we first neglect the relatively unimportant effects: those of mass loss, the resistance of phase change, and the time derivative of the interface concentration $(E=Q=N=0)$. In this case, equation (4.3) reduces to a linear equation for $\omega$, which takes the form

$$
\omega=\frac{M k^{2}}{k_{0}^{2}+k^{2}}-\frac{\bar{h}^{3} k^{2}}{3}\left(G+S k^{2}\right),
$$

with

$$
\begin{aligned}
M & =\frac{M a \Pi^{3}\left(1-\bar{c}_{I}\right)\left(c_{w}-\bar{c}_{I}\right)^{2}}{\bar{h}} \\
k_{0}^{2} & =\frac{2 \Pi^{2}\left(1-\bar{c}_{I}\right)\left(c_{w}-\bar{c}_{I}\right)}{\bar{h}^{2}}+\frac{2}{\Delta^{2}} \log \left(\frac{1-c_{0}}{1-\bar{c}_{I}}\right) \\
& =\frac{2}{\Delta^{2}} \log \left(\frac{1-c_{0}}{1-\bar{c}_{I}}\right)\left\{\frac{1-\bar{c}_{I}}{c_{w}-\bar{c}_{I}} \log \left(\frac{1-c_{0}}{1-\bar{c}_{I}}\right)+1\right\} .
\end{aligned}
$$

In the second equality of (4.6) the basic film thickness has been eliminated using (3.4). In (4.4) $k^{2}$ in the denominator of the first term in the right hand side emerges from the diffusion term in the horizontal direction of the mass transport equation. It follows that the effect of vapour diffusion along the interface mitigates the Marangoni effect 
for large wavenumbers. This is physically obvious because the former counteracts the fluctuations of the vapour concentration along the interface, which is related with the interface temperature through (2.18), and this effect is stronger for short-wavelength variations of the concentration. In (4.6) the first and second terms in the curly brackets arise from the advection and diffusion terms in the vertical direction of the mass transport equation. If the concentration difference "across the film" $c_{w}-\bar{c}_{I}$ is much less than one, the latter term can be neglected. This suggests that the variation of the vertical advection term at the interface is much larger than that of the vertical diffusion term. As a result, the dispersion relation (4.4) becomes independent of the details of the model of the latter term (2.24). Note that if the effect of the horizontal momentum diffusion is taken into account, the variation of the vertical diffusion along the interface must be weakened. If the horizontal diffusion term is also neglected, which is the case in the longwavelength limit $(k \rightarrow 0)$, the only term which contributes to the Marangoni term in the dispersion relation (4.4) is the vertical advection term. In this case, the model equation for the surface concentration (2.34) becomes equivalent to the condition that the mass flux is constant throughout the surface. Nevertheless, owing to the fluctuations of the film height and vapour concentration, the temperature also varies along the interface: thus the Marangoni effect appears. It is the presence of the vapour diffusion along the interface that locally varies the evaporation mass flux. Since the heat flux at the wall is related with the mass flux (see (2.27)), the heat transfer characteristic would also change once the instability occurs and the horizontal diffusion of the vapour takes effect.

The dispersion relation of the type of equation (4.4) was already encountered and investigated in the study of evaporating or condensing bilayer systems (Kanatani 2010). It is obvious from (4.4) that the small-wavenumber modes always grow if gravity is absent $(G=0)$. The condition that the film is stabilised by gravity reads

$$
G \bar{h}^{3} k_{0}^{2}>3 M
$$

or

$$
\frac{2 G \Delta^{2} \Pi\left(c_{w}-\bar{c}_{I}\right)}{3 M a}\left\{\log \left(\frac{1-c_{0}}{1-\bar{c}_{I}}\right)+\frac{c_{w}-\bar{c}_{I}}{1-\bar{c}_{I}}\right\}>\left\{\log \left(\frac{1-c_{0}}{1-\bar{c}_{I}}\right)\right\}^{3},
$$

where we have substituted (3.4), (4.5), and (4.6). Since the concentration difference across the film $c_{w}-\bar{c}_{I}$ is a small quantity, we expand the logarithmic function in (4.8) into a Taylor series around the wall concentration $c_{w}$ and take the terms up to the linear order to give

$$
\frac{2 G \Delta^{2} \Pi\left(c_{w}-\bar{c}_{I}\right)}{3 M a}>\left\{\log \left(\frac{1-c_{0}}{1-c_{w}}\right)\right\}^{2}-3 \frac{c_{w}-\bar{c}_{I}}{1-c_{w}} \log \left(\frac{1-c_{0}}{1-c_{w}}\right) .
$$

From (3.4) and (4.9) the linear expression for the critical thickness below which the film is destabilised is found as

$$
\bar{h}_{c}=\frac{\Delta \Pi \log \left(\frac{1-c_{0}}{1-c_{w}}\right)}{\frac{2 G \Delta^{2} \Pi}{3 M a}+\frac{3}{1-c_{w}} \log \left(\frac{1-c_{0}}{1-c_{w}}\right)} .
$$

Here the critical thickness remains finite in the limit $G \rightarrow 0$. However, this never indicates the existence of the critical thickness in the gravity-free case: this spurious behaviour emerges from linearisation.

In figure 3, the numerical values of the critical thickness obtained from (4.8) and (3.4), and the linearised expression (4.10) are plotted as functions of the wall temperature for 


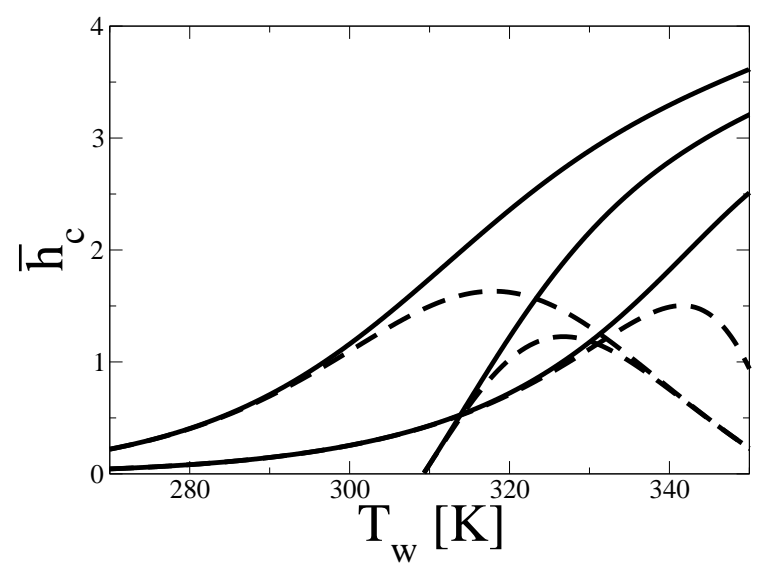

FiguRE 3 . The critical thickness $\bar{h}_{c}$ as a function of the wall temperature $T_{w}$ for the ethanol-nitrogen system of $d=0.1 \mathrm{~mm}$. The solid curves have been calculated from (3.4) and (4.8). The dashed curves represent the linearised expression (4.10). The upper and lower curves extended from lower temperatures are for $\delta=1 \mathrm{~mm}$ and $5 \mathrm{~mm}$, and $c_{0}=0$. The curves emerging at $T_{w} \simeq 310 \mathrm{~K}$ are for $\delta=1 \mathrm{~mm}$ and $c_{0}=0.2$.

the ethanol-nitrogen system with the data shown in table 1, which are assumed to be constant against the temperature variation for simplicity. The linearised expression is found to be a good approximation for small critical thicknesses. However, the two curves start to deviate at some point due to the nonlinearity of the concentration difference in (4.8). The point from which the curves of $\delta=1 \mathrm{~mm}$ and $c_{0}=0.2$ emerge corresponds to the point where the ambient concentration is equal to the wall concentration; below this temperature the condensation starts.

The expressions for the cutoff wavenumber $k_{c}$ and the maximum growth wavenumber $k_{\max }$ are obtained from (4.4) as

$$
\begin{aligned}
k_{c}^{2} & =\left(\frac{k_{0}^{2}}{2}+b\right)\left\{-1+\sqrt{1+2 \frac{a-b k_{0}^{2}}{\left(k_{0}^{2} / 2+b\right)^{2}}}\right\} \\
k_{\max }^{2} & =-\frac{2 k_{0}^{2}+b}{3}+\left[\frac{\left(k_{0}^{2}-b\right)^{3}}{27}+\frac{a k_{0}^{2}}{2}+\frac{1}{6} \sqrt{a k_{0}^{2}\left\{\frac{4}{3}\left(k_{0}^{2}-b\right)^{3}+9 a k_{0}^{2}\right\}}\right]^{1 / 3} \\
& +\left[\frac{\left(k_{0}^{2}-b\right)^{3}}{27}+\frac{a k_{0}^{2}}{2}-\frac{1}{6} \sqrt{a k_{0}^{2}\left\{\frac{4}{3}\left(k_{0}^{2}-b\right)^{3}+9 a k_{0}^{2}\right\}}\right]^{1 / 3} .
\end{aligned}
$$

where

$$
a=\frac{3 M}{2 S \bar{h}^{3}}, \quad b=\frac{G}{2 S} .
$$

Note that both the cutoff and maximum growth wavenumbers are functions of the basic film thickness. Figure 4 shows the relationship between the cutoff and maximum growth wavenumbers and the basic film thickness, calculated from (4.11) and (4.12), for the ethanol-nitrogen system. Both cutoff and maximum growth wavenumbers increase as the basic film thickness decreases from the critical thickness, and diverge at zero film thickness. This divergent behaviour already appeared in the study of the Marangoni 


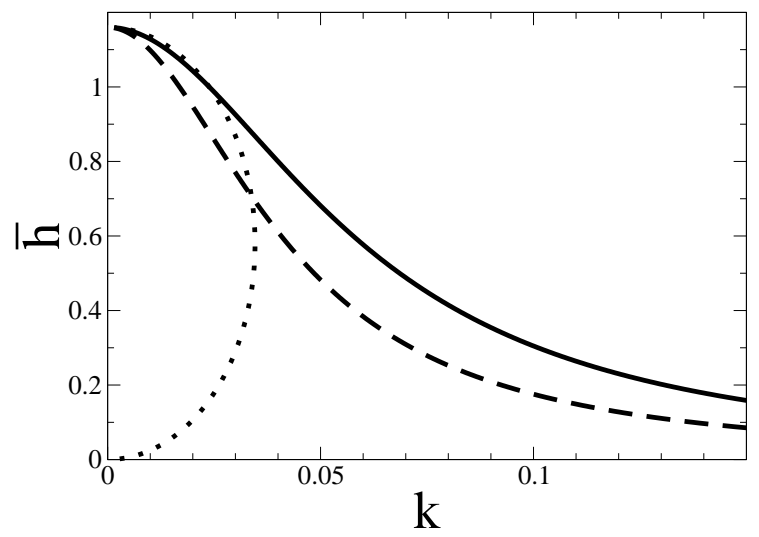

FIGURE 4 . The cutoff wavenumber $k_{c}$ (solid), the maximum growth wavenumber $k_{\max }$ (dashed), and the product of the cutoff wavenumber and the basic film thickness $k_{c} \bar{h}$ (dotted) versus the basic film thickness $\bar{h}$ for the ethanol-nitrogen system of $d=0.1 \mathrm{~mm}, \delta=1 \mathrm{~mm}, c_{0}=0$ and $T_{w}=300 \mathrm{~K}$.

dropwise condensation (Kanatani 2013). From (4.11) and (4.12) it can be deduced that the cutoff and maximum growth wavenumbers diverge as the inverse of the square root of the basic film thickness in the limit $\bar{h} \rightarrow 0$. As pointed out by Kanatani (2013), the product of the cutoff wavenumber and the basic film thickness $k_{c} \bar{h}$, which represents the cutoff wavenumber relative to the film thickness, vanishes in the limit $\bar{h} \rightarrow 0$ (see the dotted curve in figure 4). Therefore, the long-wave nature of the instability of the liquid film is preserved even though the absolute value of the cutoff wavenumber diverges as the film thickness tends to zero.

\subsection{Case $E \neq 0$ and $Q=N=0$}

Next we take into account the effect of mass loss $(E \neq 0$ and $Q=N=0)$. The dispersion relation has an additional term due to the effect of mass loss of the liquid film:

$$
\omega=\frac{M k^{2}}{k_{0}^{2}+k^{2}}-\frac{\bar{h}^{3} k^{2}}{3}\left(G+S k^{2}\right)+\frac{E \Pi\left(c_{w}-\bar{c}_{I}\right)}{\bar{h}^{2}\left(k_{0}^{2}+k^{2}\right)}\left\{\frac{2}{\Delta^{2}} \log \left(\frac{1-c_{0}}{1-\bar{c}_{I}}\right)+k^{2}\right\} .
$$

The dependence on the wavenumber of the last term is attributed to the horizontal diffusion of the vapour. Since

$$
k_{0}^{2}-\frac{2}{\Delta^{2}} \log \left(\frac{1-c_{0}}{1-\bar{c}_{I}}\right)=\frac{2 \Pi^{2}\left(1-\bar{c}_{I}\right)\left(c_{w}-\bar{c}_{I}\right)}{\bar{h}^{2}}>0
$$

the additional term is a monotonically increasing function of the wavenumber. Therefore, the destabilised effect of mass loss is enhanced for large wavenumbers because of the vapour diffusion along the interface. This fact is interpreted as follows. As shown in $\S 4.1$, the evaporation mass flux is almost constant for sufficiently small concentration differences across the film (or film thicknesses) and wavenumbers, even though the film height and vapour concentration at the interface locally vary. However, for disturbances of short wavelengths the horizontal vapour diffusion homogenises the vapour concentration along the interface. Accordingly, also the interface temperature becomes almost constant from the condition of local thermodynamic equilibrium. In this case, the temperature 


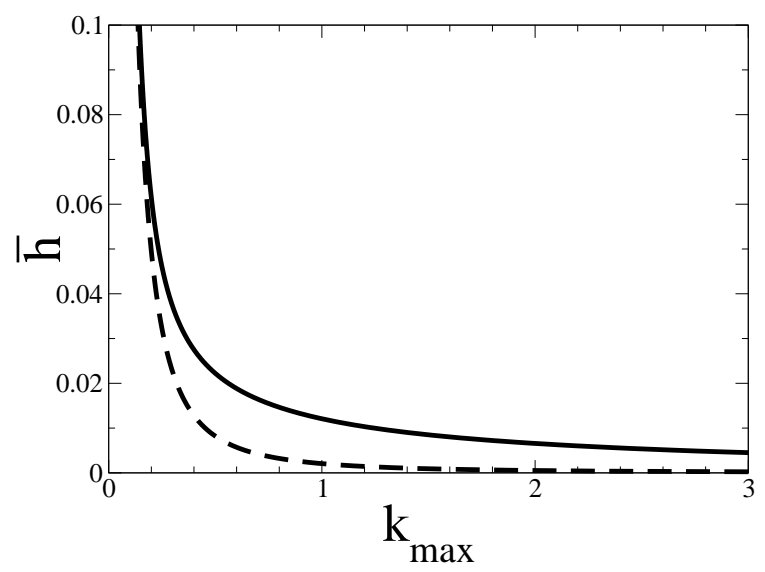

Figure 5. The maximum growth wavenumber $k_{\max }$ for $E \neq 0$ (solid) and $E=0$ (dashed) versus the basic film thickness $\bar{h}$ for the ethanol-nitrogen system of $d=0.1 \mathrm{~mm}, \delta=1 \mathrm{~mm}, c_{0}=0$, and $T_{w}=300 \mathrm{~K}$.

gradient across the film is larger for a thinner part of the film. Since the evaporation mass flux is proportional to the temperature gradient (see (2.10)), the amount of mass loss increases for the thinner portion and the deviation from the flat interface is amplified.

The maximum growth wavenumber is also calculated from (4.14) as before. Here we do not address the cutoff wavenumber because there is the ambiguity in its definition associated with the finite growth rate at $k=0$, which results from the frozen-time approach. Only the change made in the expression (4.12) to take into account the effect of mass loss is $a k_{0}^{2} \rightarrow a\left\{k_{0}^{2}+2 E /\left(M a \bar{h}^{3}\right)\right\}$. The maximum growth wavenumber for $E \neq 0$ and $E=0$ is shown in figure 5 for the ethanol-nitrogen system. The significant difference in the maximum growth wavenumber is seen for film thicknesses below $2 \mu \mathrm{m}$.

The maximum growth rate $\omega_{\max }$ for $E \neq 0$ and $E=0$ is also plotted in figure 6 . The large difference is found below $10 \mu \mathrm{m}$ of the film thickness. The maximum growth rate for $E=0$ tends to zero in the limit $\bar{h} \rightarrow 0$ whereas that for $E \neq 0$ diverges. This result implies that the effect of mass loss dominates over the Marangoni effect below $10 \mu \mathrm{m}$ thickness and the former is the primary instability mechanism. Since the time scale is proportional to the square of the length scale (see §2.2), the dependence of the growth rate on the length scale can be eliminated by multiplying the growth rate by the square of the basic film thickness. In figure 6 , we have also displayed the value of $\omega_{\max } \bar{h}^{2}$ for $E \neq 0$, which vanishes at zero film thickness. It follows that the divergent behaviour of the maximum growth rate for $E \neq 0$ never contradicts the assumption of the long-wave approximation. The growth rate at $k=0$, which represents the evolution of the basic film thickness, has been also shown in figure 6 . It is confirmed that the evolution of the disturbance is much faster than that of the basic film thickness.

$$
\text { 4.3. Case } E \neq 0, Q \neq 0 \text {, and } N=0
$$

In the previous subsections we set $Q=0$, which corresponds to the condition of local thermodynamic equilibrium at the interface. In this subsection we relax this condition and investigate an effect of the finiteness of $Q$. The expression for the growth rate for 


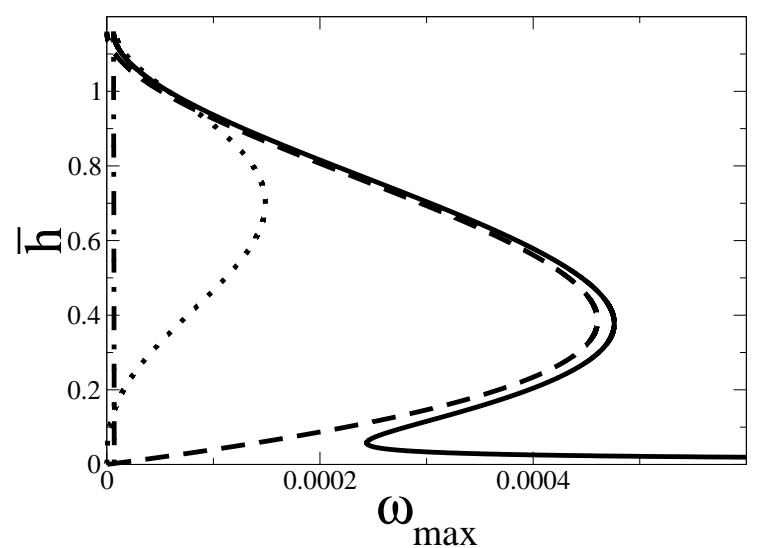

FiguRE 6 . The maximum growth rate $\omega_{\max }$ for $E \neq 0$ (solid) and $E=0$ (dashed), the product of the maximum growth rate and the square of the basic film thickness $\omega_{\max } \bar{h}^{2}$ for $E \neq 0$ (dotted), and the growth rate at $k=0$ (dot-dashed) versus the basic film thickness $\bar{h}$ for the ethanol-nitrogen system of $d=0.1 \mathrm{~mm}, \delta=1 \mathrm{~mm}, c_{0}=0$, and $T_{w}=300 \mathrm{~K}$.

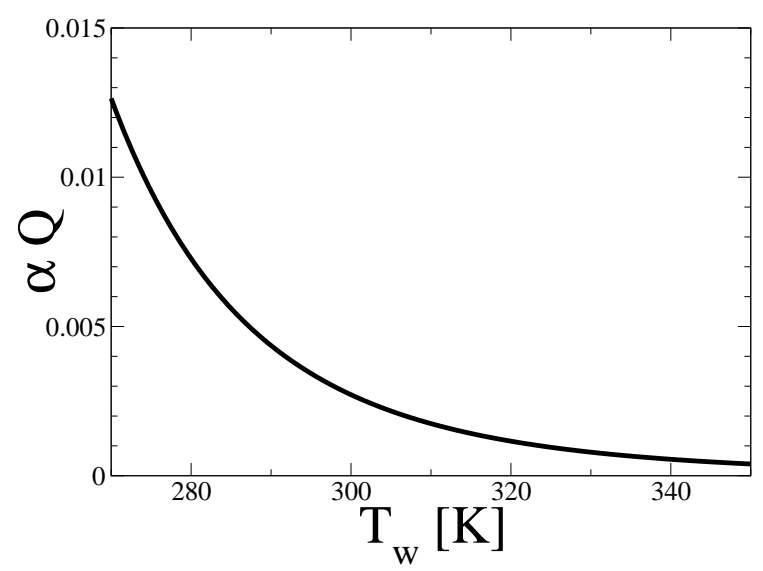

Figure 7. The temperature dependence of $\alpha Q$ for the ethanol-nitrogen system of $d=0.1 \mathrm{~mm}$.

$Q \neq 0$ becomes

$$
\begin{aligned}
\omega= & \frac{M k^{2}}{k_{0}^{2}+k^{2}}-\frac{\bar{h}^{3} k^{2}}{3}\left(G+S k^{2}\right)+\frac{E \Pi\left(c_{w}-\bar{c}_{I}\right)}{(\bar{h}+Q)^{2}\left(k_{0}^{2}+k^{2}\right)}\left\{\frac{2}{\Delta^{2}} \log \left(\frac{1-c_{0}}{1-\bar{c}_{I}}\right)+k^{2}\right\} \\
& +\frac{M a \Pi Q \bar{h}^{2}\left(c_{w}-\bar{c}_{I}\right) k^{2}}{2(\bar{h}+Q)^{2}},
\end{aligned}
$$




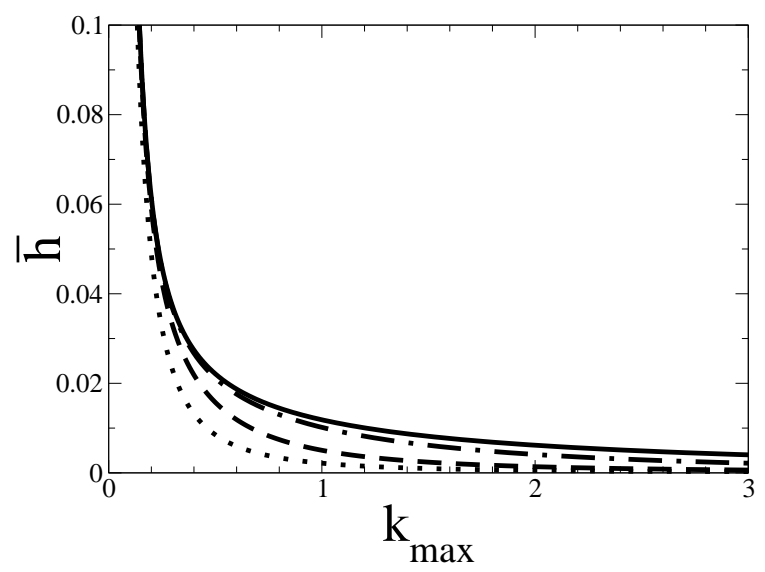

Figure 8. The maximum growth wavenumber $k_{\max }$ for $\alpha=1$ (solid), $\alpha=0.1$ (dot-dashed), $\alpha=0.01$ (dashed), and $\alpha=0.001$ (dotted) versus the basic film thickness $\bar{h}$ for the ethanol-nitrogen system of $d=0.1 \mathrm{~mm}, \delta=1 \mathrm{~mm}, c_{0}=0$, and $T_{w}=300 \mathrm{~K}$.

where

$$
\begin{aligned}
M & =\frac{M a \Pi^{3} \bar{h}^{3}\left(1-\bar{c}_{I}\right)\left(c_{w}-\bar{c}_{I}\right)^{2}}{(\bar{h}+Q)^{4}}, \\
k_{0}^{2} & =\frac{2 \Pi^{2}\left(1-\bar{c}_{I}\right)\left(c_{w}-\bar{c}_{I}\right)}{(\bar{h}+Q)^{2}}+\frac{2}{\Delta^{2}} \log \left(\frac{1-c_{0}}{1-\bar{c}_{I}}\right) \\
& =\frac{2}{\Delta^{2}} \log \left(\frac{1-c_{0}}{1-\bar{c}_{I}}\right)\left\{\frac{1-\bar{c}_{I}}{c_{w}-\bar{c}_{I}} \log \left(\frac{1-c_{0}}{1-\bar{c}_{I}}\right)+1\right\} .
\end{aligned}
$$

Note that the expression for $k_{0}^{2}$ remains unchanged if we substitute (3.4). Since $Q$, as well as $\Pi$, is inversely proportional to the saturation vapour pressure (see (2.26)), the value of $Q$ largely increases as the wall temperature decreases, as shown in figure 7 . The maximum growth wavenumber and its rate for various accommodation coefficients are plotted in figures 8 and 9 for the ethanol-nitrogen system. Here the maximum growth wavenumber is calculated in the same way as in $\S 4.2$ by replacing $G$ with $G-3 M a \Pi Q\left(c_{w}-\right.$ $\left.\bar{c}_{I}\right) /\left\{2 \bar{h}(\bar{h}+Q)^{2}\right\}$. The difference in the maximum growth wavenumber is found in a very thin region (below $2 \mu \mathrm{m}$ ), where the effect of mass loss is dominant. The maximum growth wavenumber for $\alpha=0.001$ deviates from that for the other cases even above $10 \mu \mathrm{m}$ (not shown). The growth rate significantly reduces for $\alpha<0.01$, which is in accordance with the condition $Q>1$ from figure 7. Because the parameter $Q$ appears only in the form $h+Q$ in the model equations (2.33) and (2.34), the effect of the finiteness of $Q$ emerges only when $Q \gtrsim h$. Sufficiently small accommodation coefficients even vary the critical thickness. Moreover, as seen in figure 9, the growth rate ceases to diverge at zero film thickness, while the maximum growth wavenumber itself diverges.

$$
\text { 4.4. Case } E \neq 0, Q \neq 0 \text {, and } N \neq 0
$$

Finally, the case $E \neq 0, Q \neq 0$, and $N \neq 0$ is examined. The origin of the term proportional to $N$ in (4.3) is the time derivative of the interface concentration in (2.34). Since $N$ is multiplied by $\omega$ in (4.3), this effect is stronger for larger growth rates and never alters the value of the cutoff wavenumber: physically the time derivative of the interface 


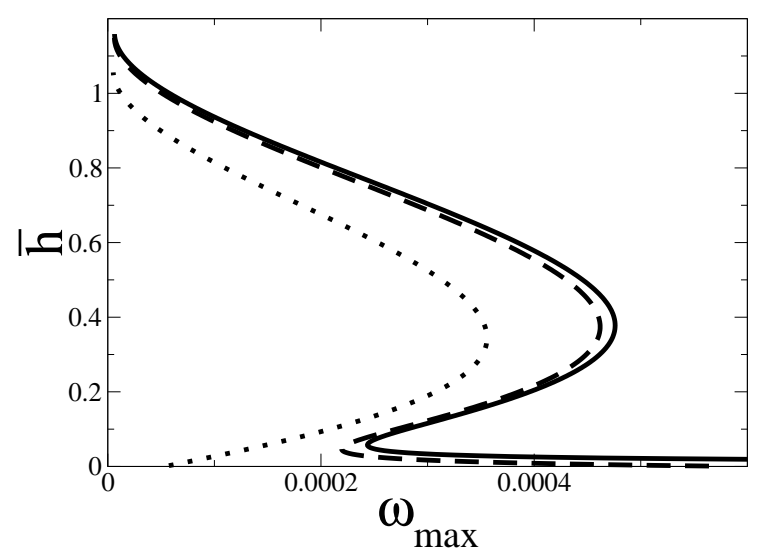

FIgURE 9. The maximum growth rate $\omega_{\max }$ for $\alpha=1$ (solid), $\alpha=0.01$ (dashed), and $\alpha=0.001$ (dotted) versus the basic film thickness $\bar{h}$ for the ethanol-nitrogen system of $d=0.1 \mathrm{~mm}, \delta=1$ $\mathrm{mm}, c_{0}=0$, and $T_{w}=300 \mathrm{~K}$.

concentration influences only rapidly growing disturbances. It is easy to show from (4.3) that this effect diminishes the Marangoni effect for $\omega>0$ :

$$
\omega=\frac{M k^{2}}{N \omega+k_{0}^{2}+k^{2}}+\cdots<\frac{M k^{2}}{k_{0}^{2}+k^{2}}+\cdots .
$$

Moreover, since $k_{0}^{2}$ becomes larger for smaller concentration differences across the film or equivalently smaller film thicknesses (see (4.6) or (4.18)), the effect of the term $N \omega$ in (4.3) appears for larger film thicknesses. Figure 10 demonstrates the difference in the growth rate between $N \neq 0$ and $N=0$ for the ethanol-nitrogen system. Here in order to accentuate the difference, we have set the wall temperature relatively higher (330 $\mathrm{K})$ and also plotted the growth rate in the gravity-free case $(G=0)$. The effect of the temporal variation of the interface concentration is observed for this system, although not so significant.

\section{Conclusions}

We have proposed the novel model of a pure liquid film evaporating into an inert gas, taking into account an effect of convection of the vapour by the evaporation flow of the gas, which was disregarded in the previous study (Sultan et al. 2005). For the liquid phase, the standard long-wave approximation is applied to the governing equations. Assuming that the variation of the vapour concentration due to the deformation of the liquid-gas interface is localised in the vicinity of the interface, we consider only the limit of the mass transport equation at the interface on the gas side. The advection term in the vertical direction of the mass transport equation at the interface is transformed into the expression in terms of the evaporation mass flux using the conservation laws of the total mass and that of one component at the interface. The vertical diffusion term is modelled by introducing the concentration boundary layer above the liquid film, on the top of which the vapour concentration is kept constant, and solving the stationary convection-diffusion equation for the concentration inside the boundary layer. Although the thickness of the boundary layer is difficult to directly measure, it is related with the 


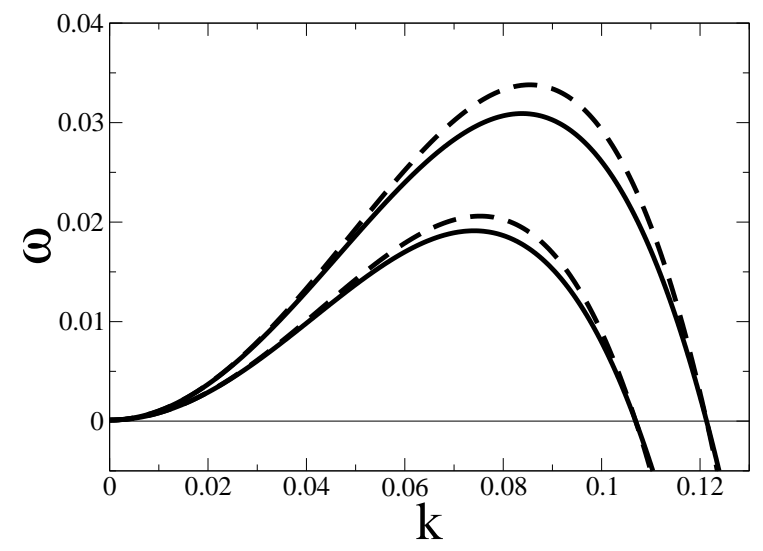

Figure 10. The growth rate $\omega$ for $N \neq 0$ (solid) and $N=0$ (dashed), and for $G \neq 0$ (lower) and $G=0$ (upper) versus the wavenumber $k$ for the ethanol-nitrogen system of $d=0.1 \mathrm{~mm}$, $\bar{h}=1, \delta=1 \mathrm{~mm}, c_{0}=0, \alpha=1$, and $T_{w}=330 \mathrm{~K}$.

heat flux on the wall, which is experimentally measurable. The two partial differential equations (2.33) and (2.34) for the two unknown variables, the film thickness and the vapour concentration at the interface, constitute our model.

The basic state is the flat-film state with the liquid height decreasing, where the vertical advection and diffusion terms of the mass transport equation balance. The linear stability analysis of the basic state with our model yields a dispersion relation essentially different from that of the previous study (Sultan et al. 2005). For simplicity we have first neglected the effects of mass loss, the resistance of phase change, and the temporal variation of the interface concentration in the mass transport equation. The effect of the vapour diffusion along the interface mitigates the Marangoni effect for short-wavelength disturbances. We have also shown that the variation of the vertical advection term can be neglected and the dispersion relation becomes independent of the details of the model of that term. In this case, the evaporation mass flux is almost constant along the interface for long-wave disturbances, whereas the effect of the horizontal vapour diffusion is responsible for the local variation of the mass flux for short-wave disturbances. In the latter case, the heat transfer characteristic at the wall would also change. From the dispersion relation, long-wavelength disturbances always grow in the gravity-free case. When gravity is present, there exists a critical thickness above which the film is stable. We have evaluated the critical thickness for the ethanol-nitrogen system (Haut \& Colinet 2005) and compared it with its linearised expression around the "wall" concentration, the vapour concentration at the wall temperature in thermodynamic equilibrium. We have obtained the analytical expressions for the cutoff and maximum growth wavenumbers, which are functions of the basic film thickness. Although the cutoff and maximum growth wavenumbers diverge at vanishing film thickness, the product of the cutoff wavenumber and the basic film thickness tends to zero, as in the study of the Marangoni dropwise condensation (Kanatani 2013).

The effect of mass loss has been also incorporated into our model. Here the term of mass loss in the dispersion relation depends on the wavenumber owing to the effect of the vapour diffusion along the interface. Since the mass flux fluctuates for short-wave disturbances as mentioned above, the destabilised effect of mass loss is intensified for 
large wavenumbers. The estimation of the maximum growth wavenumber and its rate for the ethanol-nitrogen system reveals that the effect of fluctuation of mass loss overcomes the Marangoni effect in a very thin region of the order of micro metre of the film thickness.

The effect of the deviation from local thermodynamic equilibrium at the liquid-vapour interface has been also examined. This effect is strongly dependent on the temperature, according to the variation of the saturation vapour pressure. The growth rate significantly reduces for sufficiently small accommodation coefficients $(\alpha<0.01$ for the ethanol-nitrogen system).

The effect of the time derivative of the vapour concentration at the interface in the model equation (2.34) emerges only for large growth rates and film thicknesses, and is negligible elsewhere.

In this paper, we have presented only the linear theory of the model. However, the fact that the maximum growth wavenumber is a function of the film thickness suggests that the actual wavenumber of a pattern appearing on a liquid film in an experiment depends on the film thickness at which nonlinearity emerges. Therefore, in order to specify the selected pattern nonlinear analysis should be conducted.

\section{REFERENCES}

Bestehorn, M. 2007 Convection in thick and in thin fluid layers with a free surface - the influence of evaporation. Eur. Phys. J. Spec. Top. 146, 391-405.

Bestehorn, M. \& Merkt, D. 2006 Regular surface patterns on Rayleigh-Taylor unstable evaporating films heated from below. Phys. Rev. Lett. 97, 127802.

Burelbach, J. P., Bankoff, S. G. \& Davis, S. H. 1988 Nonlinear stability of evaporating/condensing liquid films. J. Fluid Mech. 195, 463-494.

Chauvet, F., Dehaeck, S. \& Colinet, P. 2012 Threshold of Bénard-Marangoni instability in drying liquid films. Europhys. Lett. 99, 34001.

Dittmar, D., Fredenhagen, A., Oei, S. B. \& Eggers, R. 2003 Interfacial tensions of ethanol-carbon dioxide and ethanol-nitrogen. dependence of the interfacial tension on the fluid density - prerequisites and physical reasoning. Chem. Eng. Sci. 58, 1223-1233.

Haut, B. \& Colinet, P. 2005 Surface-tension-driven instabilities of a pure liquid layer evaporating into an inert gas. J. Colloid Interface Sci. 285, 296-305.

KANATANI, K. 2010 Interfacial instability induced by lateral vapor pressure fluctuation in bounded thin liquid-vapor layers. Phys. Fluids 22, 012101.

Kanatani, K. 2013 Stability of a condensing liquid film in a binary vapor mixture system. Int. J. Heat Mass Transfer 58, 413-419.

Kanatani, K. \& Oron, A. 2011 Nonlinear dynamics of confined thin liquid-vapor bilayer systems with phase change. Phys. Fluids 23, 032102.

Kimball, J. T., Hermanson, J. C. \& Allen, J. S. 2012 Experimental investigation of convective structure evolution and heat transfer in quasi-steady evaporating liquid films. Phys. Fluids 24, 052102.

LiU, R. \& Kabov, O. A. 2012 Instabilities in a horizontal liquid layer in cocurrent gas flow with an evaporating interface. Phys. Rev. E 85, 066305.

Mancini, H. \& MAZA, D. 2004 Pattern formation without heating in an evaporative convection experiment. Europhys. Lett. 66, 812-818.

Merkt, D. \& Bestehorn, M. 2003 Bénard-Marangoni convection in a strongly evaporating fluid. Physica D 185, 196-208.

Mirkovich, V. V. \& Missen, R. W. 1961 Non-filmwise condensation of binary vapors of miscible liquids. Canadian J. Chem. Eng. 39, 86-87.

Oron, A. 2000 Three-dimensional nonlinear dynamics of thin liquid films. Phys. Rev. Lett. 85, $2108-2111$.

Oron, A., Davis, S. H. \& Bankoff, S. G. 1997 Long-scale evolution of thin liquid films. Rev. Mod. Phys. 69, 931-980. 
PAlmer, H. J. 1976 The hydrodynamic stability of rapidly evaporating liquids at reduced pressure. J. Fluid Mech. 75, 487-511.

Scheid, B., Margerit, J., Iorio, C. S., Joannes, L., Heraud, M., Queeckers, P., Dauby, P. C. \& Colinet, P. 2012 Onset of thermal ripples at the interface of an evaporating liquid under a flow of inert gas. Exp. Fluids 52, 1107-1119.

Schwartz, L. W., Roy, R. V., Eley, R. R. \& Petrash, S. 2001 Dewetting patterns in a drying liquid film. J. Colloid Interface Sci. 234, 363-374.

Sultan, E., Boudaoud, A. \& Ben Amar, M. 2005 Evaporation of a thin film: diffusion of the vapour and Marangoni instabilities. J. Fluid Mech. 543, 183-202.

Thiele, U. 2010 Thin film evolution equations from (evaporating) dewetting liquid layers to epitaxial growth. J. Phys. Condens. Matter 22, 084019. 